\title{
Epistemology Probabilized
}

\author{
Richard Jeffrey
}

April 2, 2002

Here is a framework for judgment in terms of a continuum of "subjective" probabilities, a framework in which probabilistic judgments need not stand on a foundation of certainties. In place of propositional data bases, this "radical" probabilism ("probabilities all the way down to the roots") envisages full or partial probability assignments to probability spaces, together with protocols for revising those assignments and their interconnections in the light of fresh empirical or logico-mathematical input. This input need not be of the limiting 0or-1 sort. Updating by ordinary conditioning is generalized (sec. 2.2) to probability kinematics, where an observation on a random variable $X$ need not single out one value, but may prompt a new probability distribution over all values of $X$.

The effect of an observation itself, apart from the influence of prior

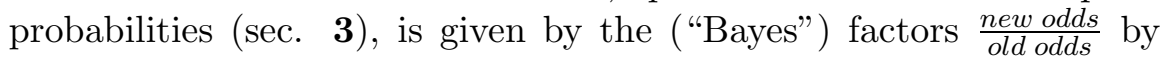
which the observer's odds between hypotheses are updated. We are not generally interested in adopting an observer's new odds as our own, for those are influenced by the observer's old odds, not ours. It is rather the observer's Bayes's factors that we need in order to use that observation in our own judgments. An account of collaborative updating is presented in these terms.

Jon Dorling's bayesian solution of the Duhem-Quine "holism" problem is sketched in sec. 4.

We finish with a brief look at the historical setting of radical probabilism (sec. 5), and an indication of how "real" probabilities can be accomodated in subjectivistic terms (sec. 6). 


\section{Judgmental ("Subjective") Probability}

Your "subjective" probability is not something fetched out of the sky on a whim; it is your actual judgment, normally representing what you think your judgment should be, even if you do not regard it as a judgment that everyone must share on pain of being wrong in one sense or another.

\subsection{Probabilities from statistics: Minimalism}

Where do probabilistic judgments come from? Statistical data are a prime source; that is the truth in frequentism. But that truth must be understood in the light of certain features of judgmental probabilizing, e.g., persistence, as you learn the relative frequency of truths in a sequence of propositions, of your judgment that they all have the same probability. That is an application of the following theorem of the probability calculus. ${ }^{1}$

Law of Little Numbers. In a finite sequence of propositions that you view as equiprobable, if you are sure that the relative frequency of truths is $p$, then your probability for each is $p$.

Then if, judging a sequence of propositions to be equiprobable, you learn the relative frequency of truths in a way that does not change your judgment of equiprobability, your probability for each proposition will agree with the relative frequency. ${ }^{2}$

The law of little numbers can be generalized to random variables:

Law of Short Run Averages. In a finite sequence of random variables for which your expections are equal, if

\footnotetext{
${ }^{1}$ See Jeffrey (1992) pp. 59-64. The name "Law of Little Numbers" is a joke, but I know of no generally understood name for the theorem. That theorem, like the next (the "Law of Short Run Averages", another joke) is quite trivial; both are immediate consequences of the linearity of the expectation operator. Chapter 2 of de Fineti (1937) is devoted to them. In chapter 3 he goes on to a mathematically deeper way of understanding the truth in frequentism, in terms of "exchangeability" of random variables (sec. 1.2, below).

${ }^{2}$ To appreciate the importance of the italicized caveat, note that if you learn the relative frequency of truths by learning which propositions in the sequence are true, and which false, then those probabilities will be zeros and ones instead of averages of those zeros and ones.
} 
you know only their arithmetical mean, then that is your expectation of each.

Then if, while requiring your final expectations for a sequence of magnitudes to be equal, you learn their mean value in a way that does not lead you to change that requirement, your expectation of each will agree with that mean. ${ }^{3}$

Example: Guessing Weight. Needing to estimate the weight of someone on the other side of a chain link fence, you select ten people on your side whom you estimate to have the same weight as that eleventh, persuade them to congregate on a platform scale, and read their total weight. If the scale reads $1080 \mathrm{lb}$., your estimate of the eleventh person's weight will be $108 \mathrm{lb}$.- -if nothing in that process has made you revise your judgment that the eleven weights are equal. ${ }^{4}$

This is a frequentism in which judgmental probabilities are seen as judgmental expectations of frequencies, and in which the Law of Little Numbers guides the recycling of observed frequencies as probabilities of unobserved instances. It is to be distinguished both from the intelligible but untenable finite frequentism that simply identifies probabilities with actual frequencies (generally, unknown) when there are only finitely many instances overall, and from the unintellible long-run frequentism that would see the observed instances as a finite fragment of an infinite sequence in which the infinitely long run inflates expectations into certainties that sweep judgmental probabilities under the endless carpet. ${ }^{5}$

\subsection{Probabilities from statistics: Exchangeability ${ }^{6}$}

On the hypotheses of (a) equiprobability and (b) certainty that the relative frequency of truths is $r$, the the Law of Little Numbers identi-

\footnotetext{
${ }^{3}$ If you learn the individual values and calculate the mean as their average without forgetting the various values, you have violated the caveat (unless it happens that all the values were the same), for what you learned will have shown you that they are not equal.

${ }^{4}$ Note that turning statistics into probabilities or expectations in this way requires neither conditioning nor Bayes's theorem, nor does it require you to have formed particular judgmental probabilities for the propositions or particular estimates for the random variables prior to learning the relative frequency or mean.

${ }^{5}$ See Jeffrey (1992) chapter 11.

${ }^{6}$ See chapters 3 and 5 of Finetti (1937), (1980).
} 
fied the probability as $r$. Stronger conclusions follow from the stronger hypothesis of

EXCHANGEABILITY: You regard propositions $H_{1}, \ldots, H_{n}$ as exchangeable when, for any particular $t$ of them, your probability that they are all true and the other $f=n-t$ false depends only on the numbers $t$ and $f .{ }^{7}$

Here, again, as in sec. 1.1, probabilities will be seen to come from statistics - but, again, only under probabilistic hypotheses.

In the presence of exchangeability of $H_{1}, \ldots H_{n}$, your probabilities for all $2^{\left(2^{n}\right)}$ of their Boolean compounds are determined by your probabilities for the following $n+1$ of those compounds:

$$
H_{t}^{n}={ }_{d f} t \text { is the number of truths among } H_{1}, \ldots, H_{n} .
$$

We can now state the following theorem. ${ }^{8}$

De Finetti's Rule of Succession. If you see $H_{1}, \ldots, H_{n}, H_{n+1}$ as exchangeable, your probability for truth of $H_{n+1}$ given a conjunction $K$ of a particular $t$ of $H_{1}, \ldots, H_{n}$ with the denials of the rest will be $P\left(H_{n+1} \mid K\right)=\frac{t+1}{n+2+*}$, where $*=(n-t+1)\left[\frac{P\left(H_{t}^{n+1}\right)}{P\left(H_{t+1}^{n+1}\right)}-1\right]$.

Example: The uniform distribution, $P\left(H_{t}^{n+1}\right)=1 /(n+2)$. In this ("Bayes-Laplace-Johnson-Carnap") case $P\left(H_{t}^{n+1}\right)=P\left(H_{t+1}^{n+1}\right)$, so that $*=0$ and de Finetti's rule says: $P\left(H_{n+1} \mid K\right)=\frac{t+1}{n+2}$.

\section{Updating on your own Observations}

In this section we determine the conditions of applicability of certain maps $P \mapsto Q$ that update your prior probability function $P$ to a posterior probability function $Q$. The maps are:

$\stackrel{A, 1}{\longmapsto}$, Conditioning on a data proposition $A$ for which $Q(A)=1$, and $\stackrel{\vec{A}, \vec{q}}{\longmapsto}$, Generalized Conditioning (or "Probability Kinematics") on a partition $A_{1}, \ldots, A_{n}$ on which you have new probabilities $Q\left(A_{i}\right)=q_{i}$.

\footnotetext{
${ }^{7}$ This comes to the same thing as invariance of your probabilities for Boolean compounds of finite numbers of the $H_{i}$ under all finite permutations of the positive integers, e.g., $P\left(H_{1} \wedge\left(H_{2} \vee \neg H_{3}\right)\right)=P\left(H_{100} \wedge\left(H_{2} \vee \neg H_{7}\right)\right)$.

${ }^{8}$ de Finett, 'Foresight', pp. 104-5. The best thing to read on this is Zabell (1989).
} 
The discussion addresses "you," a medical oncologist who is also a histo-pathologist. The $A_{i}$ are diagnoses, whose probabilities have been driven to new values $q_{i}$ by your observations. The $B_{s}$ are prognoses, "s-year survival". Your problem is to determine your new probabilities for them.

On the basis of a microscopic examination of cells wiped from your patient's bronchial tumor you have updated your probabilities on a set of mutually exclusive, collectively exhaustive diagnoses $A_{i}$ :

$$
A_{1} \text {, Benign; } A_{2} \text {, Non-small call ca.; } A_{3} \text {, Small cell ca. }
$$

How are you to extend the map $P\left(A_{i}\right) \mapsto Q\left(A_{i}\right)$ on the diagnoses to a map $P\left(B_{s}\right) \mapsto Q\left(B_{s}\right)$ on the prognoses? There is no general answer, but there are answers in the two special cases defined above: $\stackrel{A, 1}{\longmapsto}$ and $\stackrel{\vec{A}, \vec{q}}{\longmapsto}$.

\subsection{A special case: Conditioning}

Suppose an observation drives your probability for some diagnosis (say, $A_{2}$ ) all the way to 1 :

$$
Q\left(A_{2}\right)=1, \text { Certainty. }
$$

Question. When is it appropriate to update by conditioning on $A_{2}$ ?

$$
P\left(B_{s}\right) \mapsto Q\left(B_{s}\right)=P\left(B_{s} \mid A_{2}\right), \text { Conditioning. }
$$

Answer. When the change $P\left(A_{2}\right) \mapsto Q\left(A_{2}\right)=1$ leaves conditional probability given truth of $A_{2}$ invariant: ${ }^{9}$

$$
Q\left(B_{s} \mid A_{2}\right)=P\left(B_{s} \mid A_{2}\right), \text { InVARIANCE. }
$$

The invariance condition is equivalent to uniformity of expansion within $A_{2}$, i.e., constancy of odds between propositions that imply $A_{2}$ :

$$
\frac{P(C)}{P(D)}=\frac{Q(C)}{Q(D)} \text { if } C \text { and } D \text { each imply } A_{2}, \text { Uniformity. }
$$

Certainty alone is not enough to license conditioning, for one observation will generally yield many new certainties, on which conditioning would lead to different updated probability functions.

\footnotetext{
${ }^{9}$ It is easy to verify that conditioning is equivalent to invariance together with certainty.
} 
EXAMPLE. Drawing a card from a well-shuffled deck, you see that it is a heart. Conditioning on that certainty, $P$ (Queen of hearts|heart) $=$ $\frac{1}{13}$. Now in seeing that the card was a heart you also saw that it was red; but conditioning on that certainty would have yielded a different value, $P$ (Queen of hearts $\mid$ red $)=\frac{1}{26}$, which does not represent your post-observation judgmental state. ${ }^{10}$

\subsection{A less special case: Probability Kinematics}

Suppose an observation changes your probability distribution over a partition of diagnoses $A_{1}, \ldots, A_{n}$, without necessarily changing any of the $P\left(A_{i}\right)$ to $Q\left(A_{i}\right)=1$.

Question. When is it appropriate to update as follows?

$$
Q(B)=\sum_{i=1}^{n} Q\left(A_{i}\right) P\left(B \mid A_{i}\right), \quad \text { Probability Kinematics }
$$

Answer. When the invariance condition holds for each of the $A_{i}$ 's:

$$
Q\left(B \mid A_{i}\right)=P\left(B \mid A_{i}\right) \text { for } i=1, \ldots, n, \text { InVARIANCE }
$$

Notes:

- By the law of total probability, $Q(B)=\sum_{i} Q\left(A_{i}\right) Q\left(B \mid A_{i}\right)$, invariance relative to all the $A_{i}$ is equivalent to probability kinematics.

- Conditioning is the special case in which some $Q\left(A_{i}\right)=1$.

- On the native ground of probability kinematics, you are your own probability meter. In the context of your prior judgments, your new observation urges new probabilities $Q\left(A_{i}\right)=q_{i}$ upon you. In the most highly prized cases, you are able to explain these urges in terms of considerations which would weigh with other experts as well. But the urge is there, nudging the needle of your inner probability meter, even in the absence of such an explanation.

\section{Collaborative Updating ${ }^{11}$}

We now move outside the native ground of probability kinematics into a region where your new $Q\left(A_{i}\right)$ 's are based on other people's observations. You are unlikely to simply adopt such alien probabilities as

\footnotetext{
${ }^{10}$ When in doubt, condition on the stronger of two propositions for which your $Q$ is 1 . This is a version of Carnap's (1950, 1962: p. 211) "Requirement of Total Evidence".

${ }^{11}$ The best further reading is Wagner (2002).
} 
your own, for they are a confusion of the bare alien observation, which you would like to use, with the alien prior judgmental state, for which you may prefer to substitute your own.

We continue in the medical setting. You are a clinical oncologist, but no longer a histopathologist. You want to make the best use you can of the observations of a histopathologist whom you have consulted.

\subsection{Adopt the Expert's New Probabilities?}

$P$ and $Q$ : Your probabilities before and after the histopathologist's observation has replaced her prior probabilities $P^{\prime}\left(A_{i}\right)$ for the diagnoses by her posterior values $Q^{\prime}\left(A_{i}\right)$.

Will you simply adopt her new probabilities for the diagnoses, setting your $Q\left(A_{i}\right)$ 's $=$ her $Q^{\prime}\left(A_{i}\right)$ 's? If so, you can update by probability kinematics even if you had no prior diagnostic opinions $P\left(A_{i}\right)$ of your own; all you need are her new $Q^{\prime}\left(A_{i}\right)$ 's and your invariant conditional prognoses $P\left(B \mid A_{i}\right)$.

Note that she may have conditional prognoses $P^{\prime}\left(B \mid A_{i}\right)$ different from yours and invariant as yours. No matter. What concern you are her diagnoses, not her prognoses.

\subsection{Dissecting out the Purely Observational Part}

But suppose you have priors $P\left(A_{i}\right)$ which you take to be well-founded, and although you have high regard for the histopathologist's ability to interpret histographic slides, you view her prior probabilities $P^{\prime}\left(A_{i}\right)$ for the various diagnoses as arbitrary and uninformed. (Perhaps she has told you that she had no prior judgment in the matter, but for the purpose of formulating her report adopted convenient flat priors, $P^{\prime}\left(A_{i}\right)=\frac{1}{n}$ for all $i$.)

Here you would like to dissect out of the histopathologist's report the components that represent what she has actually seen, and combine them with your own priors. These components will be her BAYES FACTORS for the diagnoses, $A_{i}$, against an arbitrary "anchor" diagnosis - say, $A_{1}:{ }^{12}$

$$
\beta_{i}^{\prime}=\frac{\text { her new odds on } A_{i} \text { against } A_{1}}{\text { her old odds on } A_{i} \text { against } A_{1}}=\frac{Q\left(A_{i}\right)}{Q\left(A_{1}\right)} / \frac{P\left(A_{i}\right)}{P\left(A_{1}\right)} .
$$

\footnotetext{
${ }^{12} \mathrm{As}$ in Schwartz et al. (1981). The choice of $A_{1}$ is arbitrary, since the ratios $\beta_{i}^{\prime}: \beta_{j}^{\prime}$ would be the same with any other $A_{k}$ as anchor $(k=2, \ldots, n)$.
} 
In general, $\beta(A: B)$ is your Bayes factor on $A$ against $B:{ }^{13}$

$$
\beta(A: B)={ }_{d f} \frac{Q(A)}{Q(B)} / \frac{P(A)}{P(B)} .
$$

For diagnoses $A_{i}, A_{j}$ we then have $\beta\left(A_{i}: A_{j}\right)=\beta_{i} / \beta_{j}$.

Your probabilities and Bayes factors are $P, Q, \beta$; the histopathologist's are $P^{\prime}, Q^{\prime}, \beta^{\prime}$.

Writing your PROBABILITY FACTOR for $A_{i}$ as

$$
\pi_{i}=d f \frac{Q\left(A_{i}\right)}{P\left(A_{i}\right)},
$$

your Bayes factor on $A_{i}$ against $A_{1}$ can be written as

$$
\beta_{i}=\frac{\pi_{i}}{\pi_{1}}
$$

It is the histopathologist's $\beta^{\prime}\left(A_{i}: A_{j}\right)$ 's that tell you what she has learned about the diagnoses from the observation itself, with her prior probabilities factored out. ${ }^{14}$ To form YOuR posterior odds in the light of her observations, you can simply multiply your prior odds on $A_{i}$ against $A_{1}$ by her Bayes factors $\beta_{i}^{\prime}$. It is straightforward to verify that you can then update your probability for a prognosis $B$ by using the formula for probability kinematics in sec. 2.2, but with your $Q\left(A_{i}\right)$ 's computed as follows from her $\beta_{i}^{\prime}$ 's and your $P\left(A_{i}\right)$ 's:

$$
Q\left(A_{i}\right)=\frac{P\left(A_{i}\right) \beta_{i}^{\prime}}{\sum_{i} P\left(A_{i}\right) \beta_{i}^{\prime}} .
$$

Note that this equation can also be written, equivalently and more straightforwardly, in terms of unanchored probability factors:

$$
Q\left(A_{i}\right)=\frac{P\left(A_{i}\right) \pi_{i}^{\prime}}{\sum_{i} P\left(A_{i}\right) \pi_{i}^{\prime}},
$$

Then the updating equations for $P\left(A_{i}\right) \mapsto Q\left(A_{i}\right)$ and $P(B) \mapsto Q(B)$ can be written in terms that are neutral on the question of which alien

\footnotetext{
${ }^{13}$ In the special case where $Q(-)=P(-\mid D)$, so that updating is by conditioning on some data proposition $D$, this Bayes factor reduces to the "likelihood ratio", $\beta(A: B)=P(D \mid A) / P(D \mid B)$.

${ }^{14}$ See Wagner (2002), sec. 4. Functions of the Bayes factors will do as well, e.g., Good's (1983) "weight of evidence", $\log \beta^{\prime}\left(A_{i}: A_{j}\right)$.
} 
factors you adopt as your own, the $\beta_{i}^{\prime}$ or the $\pi_{i}^{\prime}$ : If all the ' $f_{i}^{\prime}$ 's stand for ' $\beta_{i}^{\prime}$ ' or all stand for ' $\pi_{i}^{\prime}$ ' and $N_{i}$ is your normalization

$$
N_{i}=\frac{f_{i}}{\sum_{i} P\left(A_{i}\right) f_{i}^{\prime}}
$$

of $f_{i}^{\prime}$, then if $Q\left(B \mid A_{i}\right)=P\left(B \mid A_{i}\right)$ the updating equations can be written simply as

$$
Q\left(A_{i}\right)=N_{i} P\left(A_{i}\right), \quad Q(B)=\sum_{i} N_{i} P\left(B \wedge A_{i}\right)
$$

\subsection{Updating Twice: Commutativity}

Here we consider the outcome of successive updating on the reports of two different experts - say, a histopathologist and a radiologist.

If you update twice, should order be irrelevant?

$$
\text { Should } \stackrel{1}{\mapsto} \stackrel{2}{\mapsto}=\stackrel{2}{\mapsto \mapsto} \mapsto
$$

The answer depends on particulars of

(1) the partitions on which $\stackrel{1}{\mapsto}$ and $\stackrel{2}{\mapsto}$ are defined;

(2) the mode of updating (by probabilities? Bayes factors?); and

(3) your starting point, $P$.

\subsubsection{Updating on alien probabilities for diagnoses}

A propos of (2), suppose you accept two new probability assignments to the same partition-first one, then another.

- Can order matter?

Certainly. Since the second assignment simply replaces the first, the result of accepting first one and then the other is the same as the result of simply accepting the second, regardless of the first.

- When is order immaterial?

When there are two partitions, and updating on the second leaves probabilities of all elements of the first unchanged. This happens when the two partitions are independent relative to $P .{ }^{15}$

\footnotetext{
${ }^{15}$ For more about this, see Diaconis and Zabell (1982), esp. 825-6.
} 


\subsubsection{Updating on alien factors for diagnoses}

In updating by Bayes or probability factors $f$ for diagnoses (see the end of 3.2), order cannot matter. ${ }^{16}$

Example 1: One partition. Adopting as your own both a pathologist's factors $f_{i}^{\prime}$ and a radiologist's factors $f_{i}^{\prime \prime}$ on the same partition-in either order - you come to the same result: your overall factors will be products $f_{i}^{\prime} f_{i}^{\prime \prime}=\beta_{i}^{\prime} \beta_{i}^{\prime \prime}=\pi_{i}^{\prime} \pi_{i}^{\prime \prime}$ of the pathologist's and radiologist's factors. Your final probabilities for the diagnoses and for the prognosis $B$ will be

$$
Q\left(A_{i}\right)=N_{i} P\left(A_{i}\right), \quad Q(B)=\sum_{i} N_{i} P\left(B \wedge A_{i}\right)
$$

where $N_{i}$ is the normalized factor you form from the alien $f_{i}^{\prime}$ and $f_{i}^{\prime \prime}$ :

$$
N_{i}=\frac{f_{i}^{\prime} f_{i}^{\prime \prime}}{\sum_{i} P\left(A_{i}\right) f_{i}^{\prime} f_{i}^{\prime \prime}}
$$

Example 2: Two updates: a pathologist's, $\stackrel{p a t h}{\longmapsto}$, with partition $\left\{A_{i}^{\prime}\right\}$ and factors $f_{i}^{\prime}(i=1, \ldots, m)$, and a radiologist's, $\stackrel{\mathrm{rad}}{\longmapsto}$, with partition $\left\{A_{j}^{\prime \prime}\right\}$ and factors $f_{j}^{\prime \prime}(j=1, \ldots, n)$. These must commute, for in either order they are equivalent to a single mapping, $\stackrel{\text { both }}{\longmapsto}$, with partition $\left\{A_{i}^{\prime} \wedge A_{j}^{\prime \prime} \mid P\left(A_{i}^{\prime} \wedge A_{j}^{\prime \prime}\right)>0\right\}$ and factors $f_{i}^{\prime} f_{j}^{\prime \prime}$. Now in terms of your normalizations

$$
N_{i, j}=\frac{f_{i}^{\prime} f_{j}^{\prime \prime}}{\sum_{i, j} P\left(A_{i}^{\prime} \wedge A_{j}^{\prime \prime}\right) f_{i}^{\prime} f_{j}^{\prime \prime}}
$$

of the alien factors, your updating equations on the partition elements $A_{i}^{\prime} \wedge A_{j}^{\prime \prime}$ can be written $Q\left(A_{i}^{\prime} \wedge A_{j}^{\prime \prime}\right)=N_{i, j} P\left(A_{i}^{\prime} \wedge A_{j}^{\prime \prime}\right)$. Then your new probability for the prognosis will be

$$
Q(B)=\sum_{i, j} N_{i, j} P\left(B \wedge A_{i}^{\prime} \wedge A_{j}^{\prime \prime}\right) .
$$

\footnotetext{
${ }^{16}$ Proofs are straightforward. See my Petrus Hispanus Lectures, II: 'Radical Probabilism', Actas da Sociedade Portuguesa da Filosofia (forthcoming; and currently available in http://www.princeton.edu/ bayesway/pu/Lisbon.pdf).
} 


\section{Dorling on the Duhem-Quine Problem}

Skeptical conclusions about the possibility of scientific hypothesistesting have been drawn from the presumed arbitrariness of answers to the question of which to give up - a theory (e.g., in $\mathbf{4 . 2}$ below, general relativity), or an auxiliary hypothesis ('The equipment was in working order') - when they jointly contradict empirical data. The problem, posed by Pierre Duhem in the first years of the 20th century, was reanimated by W.V. Quine in mid-century. ${ }^{17}$ But the holistic conclusion depends on the assumption that deductive logic is our only tool for confronting theories with empirical data. That would leave things pretty much as Descartes saw them, just before the mid-17th century emergence of the probabilistic ("Bayesian") methodology that Jon Dorling has brought to bear on various episodes in the history of science. Here is an introduction to Dorling's work, using extracts from his important but still unpublished 1982 paper $^{18}$

\subsection{Setting the Stage}

Were, as in Dorling's analysis, updating is by conditioning on a data statement $D$, the Bayes factor for a theory $T$ against an alternative theory $S$ equals the likelihood ratio,

$$
\beta(T: S)=\frac{P(D \mid T)}{P(D \mid S)}
$$

The empirical result $D$ is not generally deducible or refutable by $T$ alone, or by $S$ alone, but in interesting cases of scientific hypothesis testing $D$ is deducible or refutable on the basis of the theory and an auxiliary hypothesis $A$ (e.g., the hypothesis that the equipment is in good working order). To simplify the analysis, Dorling makes an assumption, prior independence, that can generally be justified by appropriate formulation of $A$ :

$$
P(A \wedge T)=P(A) P(T), P(A \wedge S)=P(A) P(S),
$$

Generally speaking, $S$ is not simply the denial of $T$, but a definite scientific theory in its own right, or a disjunction of such theories, all of

\footnotetext{
${ }^{17}$ Quine (1953), p. 41: 'our statements about the external world face the tribunal of sense experience not individually but as a corporate body.'

${ }^{18}$ This section is based on Dorling (1982). His work is also discussed in Howson and Urbach (1993). See also Dorling (1979) and Redhead (1980).
} 
which agree on the phenomenon of interest, so that, as an explanation of that phenomenon, $S$ is a rival to $T$. In any case Dorling uses the independence assumption to expand the right-hand side of the Bayes Factor $=$ Likelihood Ratio equation:

$$
\beta(T: S)=\frac{P(D \mid T \wedge A) P(A)+P(D \mid T \wedge \neg A) P(\neg A)}{P(D \mid S \wedge A) P(A)+P(D \mid S \wedge \neg A) P(\neg A)}
$$

To study the effect of $D$ on $A$, he also expands $\beta(A: \neg A)$ with respect to $T$ (and similarly with respect to $S$, although we do not show that here):

$$
\beta(A: \neg A)=\frac{P(D \mid A \wedge T) P(T)+P(D \mid A \wedge \neg T) P(\neg T)}{P(D \mid \neg A \wedge T) P(T)+P(D \mid \neg A \wedge \neg T) P(\neg T)}
$$

\subsection{Einstein/Newton, 1919}

In these terms Dorling analyzes two famous tests that were duplicated, with apparatus differing in seemingly unimportant ways, with conflicting results: one of the duplicates confirmed $T$ against $S$, the other confirmed $S$ against $T$. Nevertheless, the scientific experts took the experiments to clearly confirm one of the rivals against the other. Dorling explains why the experts were right:

"In the solar eclipse experiments of 1919, the telescopic observations were made in two locations, but only in one location was the weather good enough to obtain easily interpretable results. Here, at Sobral, there were two telescopes: one, the one we hear about, confirmed Einstein; the other, in fact the slightly larger one, confirmed Newton. Conclusion: Einstein was vindicated, and the results with the larger telescope were rejected." (1982, sec. 4)

Notation

$T$ : Einstein: light-bending effect of the sun

$S$ : Newton: no light-bending effect of the sun

$A$ : Both telescopes are working correctly

$D$ : The conflicting data from both telescopes

In the Bayes factor $\beta(T: S)$ above, $P(D \mid T \wedge A)=P(D \mid S \wedge A)=0$ since if both telescopes were working correctly they would not have given contradictory results. Then the first terms of the sums in numerator and denominator vanish, so that the factors $P(\neg A)$ cancel and we have

$$
\beta(T, S)=\frac{P(D \mid T \wedge \neg A)}{P(D \mid S \wedge \neg A)}
$$


Dorling continues: "Now the experimenters argued that one way in which A might easily be false was if the mirror of one or the other of the telescopes had distorted in the heat, and this was much more likely to have happened with the larger mirror belonging to the telescope which confirmed $S$ than with the smaller mirror belonging to the telescope which confirmed $T$. Now the effect of mirror distortion of the kind envisaged would be to shift the recorded images of the stars from the positions predicted by $T$ to or beyond those predicted by $S$. Hence $P(D \mid T \wedge \neg A)$ was regarded as having an appreciable value, while, since it was very hard to think of any similar effect which could have shifted the positions of the stars in the other telescope from those predicted by $S$ to those predicted by $T, P(D \mid S \wedge \neg A)$ was regarded as negligibly small, hence the result as overall a decisive confirmation of $T$ and refutation of $S . "$ Thus the Bayes factor $\beta(T, S)$ is very much greater than 1 .

\section{Radical Probabilism}

Descartes sought to refute skepticism about experience by proving the existence of a God $\left(={ }_{d f}\right.$ the perfect being) who surely does not deceive us. On this "dogmatic" foundation of certainty he would build empirical science.

Shortly after Descartes's death, probabilistic thinking - in much the same form in which we know it today-emerged from a famous correspondence between Fermat and Pascal. It was given the place of honor at the end of the best-selling "How to Think" book known as "The Port-Royal Logic" (Arnauld, 1662):

"To judge what one must do to obtain a good or avoid an evil, it is necessary to consider not only the good and the evil in itself, but also the probability that it happens or does not happen; and to view geometrically the proportion that all these things have together."

But a quasi-Cartesian dogmatism figured prominently in 20th century thought about the foundations of probabilistic thinking. Here is an example from the pragmatist-empiricist philosopher C. I. Lewis (1946, p. 186):

"If anything is to be probable, then something must be certain. The data which themselves support a genuine prob- 
ability, must themselves be certainties. We do have such absolute certainties, in the sense data initiating belief and in those passages of experience which later may confirm it."

What I call "radical probabilism" denies this claim. The claim itself seems to be based on the thought that conditioning on certainties is the only way to update probabilities. That basis for dogmatic probabilism is undermined by the existence of a generalized conditioningprobability kinematics - as a way of updating on mere probabilities.

Radical probabilism offers Bayes factors as a surrogate for Lewis's absolute certainties. Lewis held that these certainties cannot

"be phrased in the language of objective statement - because what can be so phrased can never be more than probable. Our sense certainties can only be formulated by the expressive use of language, in which what is signified is a content of experience and what is asserted is the givenness of this content."

In radical probabilism the Bayes factors $\beta_{i j}$ do the job of your ineffable sense certainties. Like those ineffables, your Bayes factors lie outside the Boolean algebra of objective statements on which your $P$ and $Q$ are defined. But where Lewis can give no intelligible account of your sense certainities, radical probabilism can identify the $\beta$ 's as ratios of your new to old odds between items that are expresible in the language of objective statement.

\section{Real Subjectivism}

Radical probabilism gets along without objective probabilities, real chances (" $R$ "). The thought is that these are nothing but projections of judgmental probabilities $P$ out into the world, whence we hear them clamoring to be let back in. The following equation ("Miller's Principle") could be their return ticket: ${ }^{19}$

$$
P[H \mid R(H)=r]=r \text { if ' } r \text { ' is a purely mathematical designator. }
$$

\footnotetext{
${ }^{19}$ Here, ' $r$ ' might be '.7' or ' $1 / \pi$ ', but not ' $1 /($ my mass in $\mathrm{Kg})$ ', and not ' $R(H)$ '. The thought is that you must be able to tell what number ' $r$ ' denotes without recourse to any empirical facts. The need for this restriction is seen when we put ' $R(H)$ ' for ' $r$ '. We then have $P[H \mid R(H)=R(H)]=R(H)$, which is equivalent to $P(H)=R(H)$. Unrestricted, Miller's Principle thus implies that your subjective
} 
(If you know the real chance, that will be your judgmental probability.) But they don't need a return ticket; they never really left.

There certainly are numbers "out there" - numbers like the fraction of 70-year-olds who live to be 80 - and it may well be that if you knew that number you would adopt it as your judgmental probability that your 70-year-old uncle, Bob, will live to be 80 . But maybe not. Maybe you know that Bob comes from remarkably long-lived stock, in which case your probability for his reaching 80 might be higher than the statistics on 70-year-olds would suggest. (But maybe you also know that Bob has pancreatic cancer, so that your probability is below the statistical average for 70-year-olds.) This is the famous reference-class problem. According to radical probabilism, this problem can be solved by putting the horse before the cart, using your probabilistic judgments to choose among the various numbers out there. But the point is that there are enough familiar numbers out there - statistics, fractions of green balls in urns, etc. - to do the jobs that objectivists send "real probabilities" out there to do.

Once such numbers have been chosen, formulas that look rather like the return ticket may come into play:

$$
P(H \mid X=x)=x \text {, where } X \text { is an ordinary random variable }
$$

ExAmple. $P$ (A green ball will be drawn $\mid 70 \%$ are green $)=70 \%$ if you think the balls are well mixed, etc.

To hypostasize $R(H)$ as a physical magnitude is to sweep the subjective element in "objective" probability under the carpet. We do better to identify the parameter $X$ case by case, as the fraction of green balls in the urn, or of septuagenerian men who live to be 80 , or whatever. In all of these quests for a suitable $X$ the great clue, the sticky subjective core, is that, whatever $X$ turns out to be, it must satisfy the equation $P(H \mid X=x)=x$.

probabilities always agree with the objective probabilities. In effect, Miller (1966) rejected the restriction and welcomed the result as a reductio ad absurdum of the concept of judgmental probability. (Note that since ' $R$ ' means real chance, not future probability, van Fraassen's (1984) reflection principle is only formally identical with Miller's principle.) 


\section{References}

Arnauld, Antoine $(1662,1964)$ Logic, or, the Art of Thinking ("The Port-Royal Logic") tr. J. Dickoff and P. James, Bobbs-Merril, Indianapolis.

Bayes, Thomas (1763) An essay towards solving a problem in the doctrine of chances, Philosophical Transactions of the Royal Society 53, $370-418$.

Carnap, R. (1950), Logical Foundations of Probability, Chicago: University of Chicago Press. (2nd ed., 1962)

de Finetti, Bruno (1937, 1980) La prévision, ses lois logiques, ses sources subjectives, Annales de l'institut Henri Poincaré 7, 1-68. Translation ("Foresight ...") in Studies in Subjective Probability," Henry Kyburg, Jr. and Howard Smokler (eds.), Krieger, New York, 53-118.

Diaconis, Persi and Sandy Zabell (1989) Updating Subjective Probability, Journal of the American Statistical Society 77, 822-30.

Dorling, Jon (1979) Bayesian personalism, the methodology of research programmes, and Duhem's problem, Studies in History and Philosophy of Science 10, 177-87.

Dorling, Jon (1982) Further illustrations of the Bayesian solution of Duhem's problem (29 pp., photocopied); see http : \\www.princeton.edu $\rceil^{\sim}$ bayesway

Duhem, Pierre (1906, 1914. 1954) La Théorie Physique: Son Object, Sa Structure, Paris. Translation, The Aim and Structure of Physical Theory. Princeton University Press.

Field, Hartry (1978) A Note on Jeffrey Conditionalization, Philosophy of Science 45, 361-7.

Good, I.J. (1983) Good Thinking, University of Minnesota Press.

Howson, Colin and Peter Urbach (1993) Scientific Reasoning: the Bayesian approach, Open Court, La Salle, Illinois, 2nd ed. 
Jeffrey, Richard (1970) [untitled review of Miller (1966) and other papers] Journal of Symbolic Logic 35, 124-127.

Jeffrey, Richard (1992) Probability and the Art of Judgment, Cambridge U. P.

Lewis, C. I. (1946) An Analysis of Knowledge and Valuation, Open Court.

Miller, David (1966) A Paradox of Information, British Journal for the Philosophy of Science 17, 59-61.

Neurath, Otto (1932), Protokollsätze, Erkenntnis 3, 204-214. Translated in his Philosophical Papers 1913-1946, Dordrecht: Reidel.

Quine, Willard van Orman (1951. 1953) Two Dogmas of Empiricism, Philosophical Review 60, 20-43. Reprinted in From a Logical Point of View, Harvard University Press.

Redhead, Michael (1980) A Bayesian reconstruction of the methodology of scientific research programmes. Studies in History and Philosophy of Science 11, 341-7.

Schwartz, W.B., Wolfe, H.J., and Pauker, S.G. (1981) Pathology and Probabilies: A New Approach to Interpreting and Reporting Biopsies, The New England Journal of Medicine 305, 917-23.

van Fraassen, Bas (1984) Belief and the will, J. Philosophy 65, 243-60.

Wagner, Carl (2002, forthcoming), Probability kinematics \& commutativity, Philosophy of Science (=http://www.princeton.edu/ bayesway/Wagner.pdf)

Zabell, Sandy (1989) The Rule of Succession. Erkenntnis 31, 283321. 